\title{
La madera deTrema micrantha (L.) Blume de Veracruz, México
}

\section{Wood of Trema micrantha (L.) Blume from Veracruz, México}

\author{
Alejandra Quintanar-Isaías¹, Marco A. Jacobo-Villa², \\ Citlalli López-Binnqüist ${ }^{3}$, Noé Flores-Hernández¹, \\ Ana Teresa Jaramillo-Pérez ${ }^{1}$ y Carmen de la Paz Pérez-Olvera1
}

\begin{abstract}
RESUMEN
Se estudió la anatomía microscópica, los índices hidráulicos y mecánicos de la madera de 28 árboles de Trema micrantha de cuatro localidades ubicadas en los municipios de Xico y Coatepec en el estado de Veracruz. Adicionalmente, se determinaron las características macroscópicas, la densidad relativa, el contenido de humedad y algunas pruebas de carpintería en probetas de diferentes tamaños de la madera de tres árboles, con el fin de proponer usos alternativos en zonas rurales. Los resultados mostraron que $T$. micrantha presenta un xilema mesomórfico, poco resistente a la cavitación, de baja densidad relativa, con valores bajos de índices de colapso de vaso y fibras y altos para el índice de vulnerabilidad. Los análisis de varianza confirman que de todas las características estudiadas solamente el índice de colapso de los vasos es similar entre indivíduos y poblaciones. Las comparaciones múltiples arrojan que los caracteres que separan a las localidades Bola de Oro y Los Barrales de las otras son el grosor de la pared y el número de radios. Las pruebas tecnológicas describen una madera que no se raja al clavado, responde bien al ensamblado y al torneado por lo que se recomienda para su uso en la carpintería rural.
\end{abstract}

PALABRAS CLAVE:

Anatomía de la madera, carpintería, densidad relativa, tecnología de la madera, zonas rurales.

\begin{abstract}
The microscopic anatomy, hydraulic and mechanical indices of the wood of 28 trees of the species Trema micrantha of four localities in the municipalities of Xico and Coatepec in Veracruz State, Mexico were studied. In addition, macroscopic characteristics such as relative density and moisture content were determined. Results showed that $T$. micrantha has a mesomorphic xylem characterized by low resistance to cavitation, low values of relative density, low indices for vessel and fiber collapse and a high index of vulnerability. An analysis of variance shows that of all the characteristics studied, only the rate of collapse of the vessels is similar between individuals and populations. Multiple comparison tests show that wall thickness and the number of rays separate populations Bola de Oro and Barrales from the other sites of study.Carpentry tests reveal that wood from T. micrantha is resistant to splitting when nailed, is well suited to assemblage, and thus, it is recommended for use in carpentry in rural areas.
\end{abstract}

KEY WORDS:

Wood anatomy, carpentry, specific gravity, wood technology, rural areas.

1 Departamento de Biología. C.e.: aqi@xanum.uam.mx.

2 Departamento de Ingeniería de Procesos e Hidráulica.

1,2 Universidad Autónoma Metropolitana. Av. San Rafael Atlixco 186, Colonia Vicentina, Iztapalapa.

3 Centro de Investigaciones Tropicales, Universidad Veracruzana. 


\section{INTRODUCCIÓN}

El género Trema se considera pantropical, contiene entre 14 y 55 especies (Vázquez-Yanes et al., 1999). T. micrantha (Ulmaceae), tiene muy amplia distribución, desde el sur de Florida, EUA hasta el norte de Argentina. En México crece desde el Sur de Tamaulipas hasta Yucatán y Quintana Roo y en la vertiente del Pacífico desde Sonora y Sinaloa hasta Chiapas. A lo largo de su distribución recibe una gran diversidad de nombres locales. En Chiapas se conoce como capulín, capulín cimarrón, colorada, pellejo de vieja; en Oaxaca como cuero; en Michoacán como equipal; en la Sierra Norte de Puebla como jonote o chacá; y en Veracruz como checait, guacimilla e ixpepe (Gutiérrez y Dorantes, 2004).

En México, los árboles de esta especie pueden encontrarse en varios tipos de vegetación, desde selvas bajas de diversa caducidad y medianas y altas perennifolias hasta bosques mesófilos de montaña (Vázquez-Yanes et al., 1999) y pueden alcanzar hasta $20 \mathrm{~m}$ de alto en 7 años, son heliófitos y de rápido crecimiento (Ackerly, 1997; Vázquez-Yanes et al., 1999). Trema micrantha produce abundante inflorescencia con pequeñas flores monoicas una vez al año, lo que explica que se considere como la segunda especie después de Ficus spp., para alimentar a una gran cantidad de aves migratorias que son vehículo de dispersión (Ackerly, 1997). Cuando los árboles crecen en bosques secundarios y llegan aproximadamente a los 30 años de edad, son reemplazados naturalmente por árboles de otros taxa más longevos.

Esta especie produce una gran cantidad de materia orgánica debido al rápido crecimiento de su follaje, lo que promueve mejores condiciones del microclima y calidad del suelo, que lo hace muy estable y con buenas condiciones de humedad (Vázquez-Yanes et al., 1999), por lo que ha sido recomendada en la reforestación de áreas perturbadas (National Academy of Sciences, 1980; Vázquez-Yanes et al., 1999). Al respecto, en Brasil, $T$. micrantha es utilizada como una de las especies clave en la recuperación de áreas erosionadas o degradadas (Vázquez-Yanes et al., 1999; Carvalho, 1994). Se considera una especie con alto valor ecológico pues constituye una fuente de alimento clave para varias especies de aves en plantíos forestales (Ferreira et al., 1976), en la preservación de áreas sujetas a inundaciones y en la estabilización de dunas (Amorim et al., 1997). Selaya et al. (2008) observaron que en sucesiones secundarias en selvas tropicales de la amazonia boliviana, $T$. micrantha es la primera en dominar la cubierta vegetal. En un estudio de dendrometría, Ferreira et al. (2009), demostraron que $T$. micrantha es una de las más productoras de hojarasca, pues encontraron que la producción de ésta se relaciona directamente con la altura y el diámetro de los troncos.

Se conoce que en países centroamericanos $T$. micrantha es subutilizada, aunque en Guatemala, esta especie es la que presenta mayor variedad de usos actuales y potenciales, tales como: pilotes, postes, madera en rollo, leña, forrajes, como sombra, para conservación de suelos y aguas e incluso para la producción de pulpa y papel (Melgar, 2003).

Gelder et al. (2006) realizaron un estudio sobre las relaciones entre la mecánica de la madera, la alometría y la historia de vida de varias especies de bosques tropicales lluviosos de Bolivia. Estos autores registraron para $T$. micrantha valores de densidad $(\mathrm{pa} / \mathrm{vm}$, $\mathrm{g} \mathrm{cm}^{-3}$ : $\mathrm{pa}=$ peso anhidro; $\mathrm{vm}=$ volumen al momento de la prueba) en estado saturado y seco para ramas $(0,75 ; 0,23$; 
respectivamente) y para tronco $(0,74$; 0,20 ; respectivamente). También determinaron valores de resistencia en flexión (Módulo de ruptura, MOR 25,1 $\mathrm{N} \mathrm{mm}-2$ ), resistencia a la compresión (11,4 $\left.\mathrm{N} \mathrm{mm}^{-2}\right)$, módulo de elasticidad (MOE $3533 \mathrm{~N} \mathrm{~mm}^{-2}$ ) y otros valores mecánicos asociados a las resistencias de las ramas en árboles en pie. De acuerdo con IAWA (1989) la densidad relativa de esta madera en seco sería ligera o baja $(\leq 0,40)$.

Williams (1992) estudió el crecimiento diamétrico de varias especies arbóreas del bosque de neblina de los alrededores de Xalapa encontrando que los de $T$. micrantha se enmarcan en la clase diamétrica $20 \mathrm{~cm}-29 \mathrm{~cm}$. Hasta entonces su madera no recibía ningún uso industrial.

Bárcenas y Ordóñez (2008) estudiaron la calidad de la madera de T. micrantha y otras especies utilizadas como sombra de cafetales en el centro de Veracruz. Los autores calificaron la madera de esta especie como con densidad relativa moderadamente ligera $(0,42)$, muy rápida para secarse, con severas deformaciones, de contracciones moderadas y de resistencia mecánica baja. Con relación al maquinado y la trabajabilidad, la sitúan como una madera fácil de trabajar pero regular para el cepillado.

Trema micrantha se ha usado para la producción de papel amate en la Sierra Norte de Puebla, en sustitución de especies de Ficus y Morus, tradicionalmente utilizadas para esta labor. T. micrantha es la única que puede descortezarse durante todo el año y es también la que crece de manera más rápida y extensa, siendo uno de los árboles preferidos para la sombra de cafetales, cercas, postes construcciones ligeras y eventualmente como leña y planta medicinal (Sangalli et al., 2002; López, 2003; Toledo y Salick, 2006; Martínez et al., 2007). Durante los últimos
30 años los cosechadores de corteza en el estado de Puebla, México, han empleado árboles de esta especie con diámetros menores a $20 \mathrm{~cm}$, pues es la edad del árbol ideal para obtener las fibras de su corteza para la elaboración del papel amate. Sin embargo, recientemente las poblaciones se han visto mermadas por una sobrexplotación de los individuos más jóvenes. Además, después de la cosecha, la madera de estos árboles queda en pie y expuesta directamente al ambiente, por lo que rápidamente pierde su valor como material maderable comercializable y sólo puede emplearse para leña o reincorporarse al suelo (Quintanar et al., 2010; López et al., 2012). Esto plantea una alternativa metodológica para la valoración tecnológica, si el fin que se persigue es buscar y promocionar nuevos usos para T. micrantha con diámetros menores a $20 \mathrm{~cm}$, como una forma opcional para la economía y subsistencia de las poblaciones rurales. Uno de los usos promisorios de la madera de esta especie es en la carpintería rural.

\section{OBJETIVOS}

Evaluar las características anatómicas e índices hidráulicos y mecánicos de la madera de árboles jóvenes de Trema micrantha, para conocer la variación entre individuos y entre localidades, con el fin de entender su respuesta tecnológica. De esta manera se pretende contribuir al conocimiento de sus características tecnológicas para considerarla como una especie potencial en la carpintería rural en las zonas rurales cafetaleras.

\section{METODOLOGÍA}

Sitios de muestreo. La madera procede de 28 árboles de Trema micrantha de los predios: El Grande (localidad 1), municipio de Coatepec; Bola de Oro (localidad 
2), Plan de Lago Preso (localidad 3) y Los Barrales (localidad 4) del municipio de Xico (Fig.1), todos en el estado de Veracruz, México. En cada predio se muestrearon siete árboles. En El Grande, el clima es templado, con temperatura promedio de $19,2^{\circ} \mathrm{C}$; precipitación pluvial media anual de $1926 \mathrm{~mm}$; lo irrigan cuatro arroyos tributarios del río de Los Pescados o La Antigua: El Pixquiac, Los Pintores, El Sordo y El Hueyapan. Bola de Oro, Plan de Lago Preso y los Barrales pertenecen al municipio de Xico, en el que el intervalo altitudinal va de 1100 msnm a $1400 \mathrm{msnm}$. Se registra un clima templado, con temperatura media anual de $19^{\circ} \mathrm{C}$; con precipitación total anual de $1200 \mathrm{~mm}$ a $1500 \mathrm{~mm}$; la temperatura del mes más frío varía entre $3{ }^{\circ} \mathrm{C}$ y $18{ }^{\circ} \mathrm{C}$ (Gómez, 1991). Los suelos que se desarrollan pueden ser de tipo ferralíticos, profundos, arcillosos y poco porosos, así como andosoles diferenciados y húmicos, profundos, muy porosos y limosos. En las barrancas profundas se avizoran afloramientos rocosos, aunque los suelos se muestran poco erosionados.

La reserva de agua más importante del centro del estado se encuentra en la franja oriental del Volcán Cofre de Perote, pues alimenta los ríos Bobo, Actopan y Los Pescados. Los ríos Calpixcan, Hueyapan, La Funda y el Matlacóbatl son los más importantes de la zona y tributarios todos ellos del río Los Pescados. El río Matlacóbatl es de los más grandes en longitud en el municipio y da origen a las cascadas La Monja y Texolo (Gómez, 1991).

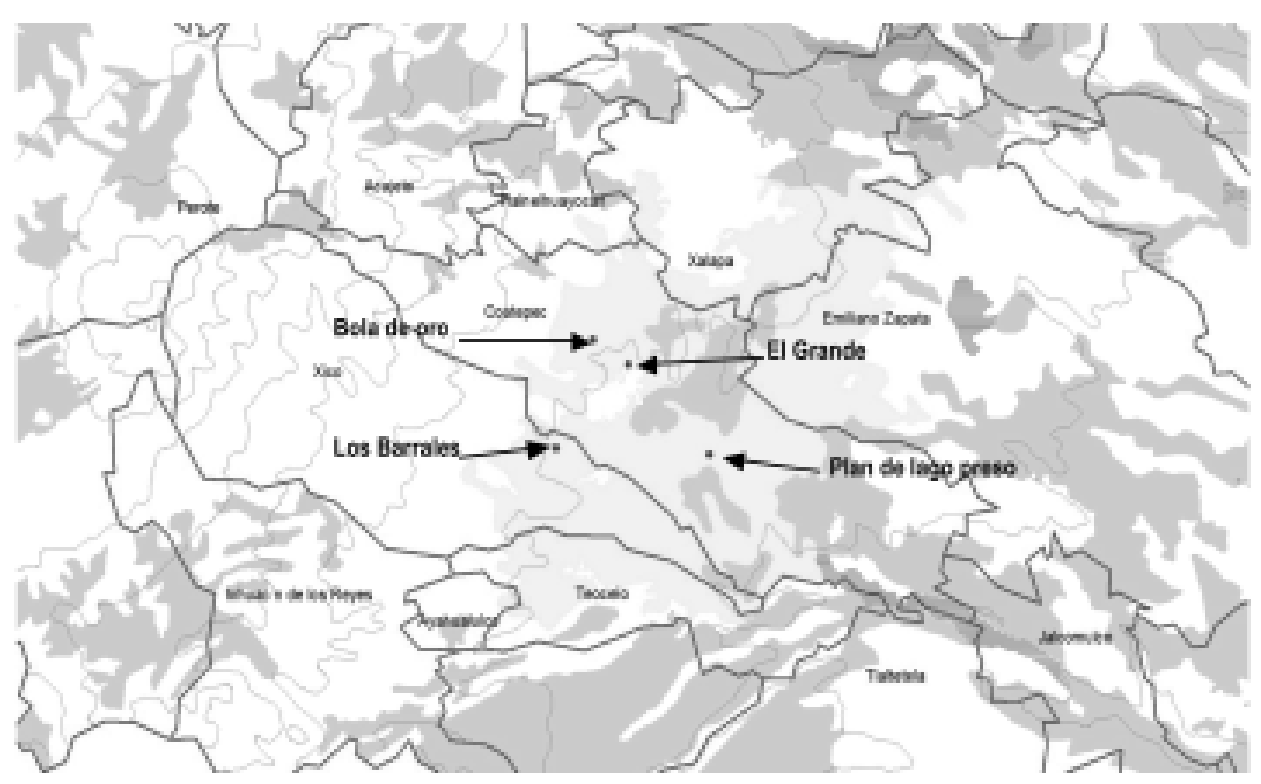

Figura 1. Sitios de colecta, municipios de Coatepec y Xico, Veracruz. 


\section{Procesamiento de material}

1. Anatomía microscópica, índices de vulnerabilidad (I.V.), de colapso (I.C.) y conductividad hidráulica teórica $\mathrm{K}_{h}$-teórica.

De los fustes de los 28 árboles seleccionados de hasta $20 \mathrm{~cm}$ de DAP, se obtuvieron piezas de madera de $5 \mathrm{~cm}$ de profundidad y $5 \mathrm{~cm}$ de ancho y largo. Estas piezas se dimensionaron para obtener tres cubos de $2 \mathrm{~cm}$ por lado. Cada cubo se ablandó usando una solución de alcohol-glicerol-agua (a.g.a.), infiltrándola al vacío durante una semana. Después, los cubos se colocaron en una solución de PEG al 5\% para elaborar cortes transversales, tangenciales $y$ radiales en un criostato marca Leica a $-20{ }^{\circ} \mathrm{C}$. Las muestras se pegaron en portaobjetos con adhesivo de Haup. Para la tinción se usó azul de toluidina, lavando los cortes y dejándolos secar. Se añadieron gotas de xilol y se montaron en resina Entellan. Adicionalmente, se preparó el material disociado de cada de cubo de acuerdo con los protocolos de Johansen (1940).

En la sección transversal se midieron: diámetro de los vasos y número por milímetro cuadrado; en la sección tangencial: altura y anchura de radios, así como número de radios por milímetro. En el material disociado se midieron longitud, grosor y diámetro de fibras y longitud de vasos. En la sección radial se midieron altura y achura de las punteaduras intervasculares. Se hicieron al menos 30 mediciones por cada carácter. La clasificación de los tamaños se hizo de acuerdo con Chattaway (1932). Los caracteres cualitativos se describieron de acuerdo con IAWA (1989). Por otro lado, se determinaron los contenidos celulares de acuerdo con Quintanar et al. (2004).

Para el cálculo del índice de vulnerabilidad (I.V.) se usó la ecuación 1 de
(Carlquist, 1977).

$$
l y_{n}=\frac{d v}{n}
$$

donde:

$d v$ : diámetro de vaso

$n$ : número de vasos $/ \mathrm{mm}^{2}$

Para calcular el índice de colapso (I.C.) se usó la ecuación 2 (Hacke et al., 2001).

$$
\text { I.C. }=\left(\frac{2 g p}{d}\right)^{2}
$$

donde:

gp: grosor de la pared de la célula (vaso o fibra)

d: diámetro de la pared de la célula (vaso o fibra)

La conductividad hidráulica $\left(k_{h}\right)$ teórica se obtuvo aplicando la ecuación 3 (Streeter y Wyllie, 1985).

$$
\frac{m}{t}=\frac{\rho m D^{4}}{128 \mu} \frac{d P}{d l}=\frac{\rho \sigma^{4}}{8 \mu} \frac{d P}{d l}=k_{k}
$$

donde:
$m: \quad$ masa del fluido
$t$ : tiempo
$d P$ : cambio de la presión
$d l$ : cambio de longitud
$D$ : diámetro de la sección
$r$ r radio de la sección
$\mu$ : viscosidad dinámica del fluido
$\rho: \quad$ densidad del fluido

Se calculó el diámetro y el radio de la sección del segmento estudiado. Con los datos de diámetro y número de vasos $/ \mathrm{mm}^{2}$, se obtuvo el área efectiva teórica de conducción para el cálculo de $\mathrm{k}_{\mathrm{h} \text {-area }} \mathrm{y}$ el diámetro equivalente del flujo 


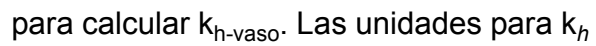
son: $\mathrm{m} \mathrm{kg} \mathrm{MPa} \mathrm{s}{ }^{-1}$ y los índices se consideran adimensionales (Quintanar, 2009).

Se estimaron las medias y las desviaciones estándar con las que se obtuvieron las categorías numéricas y la clasificación de las características anatómicas mensurables. Para evaluar las diferencias de estás últimas, se realizaron análisis de varianza (ANOVA) de $\mathrm{K}_{h}$, I.V., I.C.vaso e I.C. .fibras, considerando a las localidades y los árboles como variables categóricas. También se analizó la correlación de Pearson de todas las variables.

Además, se hizo la prueba de comparación múltiple Tukey-Kramer $(p<0,05)$ para separar a las localidades considerando a los caracteres anatómicos y un análisis discriminante, con el propósito de detectar diferencias de los caracteres microscópicos mensurables en los siguientes niveles: 1) entre árboles y 2) entre localidades. Se realizaron análisis discriminantes múltiples (ADM) sobre una matriz de 699 muestras, 28 árboles y 10 variables anatómicas. El criterio utilizado para evaluar las diferencias entre los grupos fue la $\lambda$ de Wilks, que tiende a tomar valores cercanos a cero cuando los grupos están bien definidos en función de las variables consideradas y tiende a uno cuando los grupos no están bien definidos (Tatsuoka, 1970). Se realizaron dos análisis discriminantes: (i) considerando a los siete árboles muestreados por localidad, haciendo un total de 28 árboles; (ii) separando a las muestras por localidades, haciendo un total de cuatro. Se extrajeron las funciones discriminantes correspondientes y se identificaron aquellas variables que más contribuyen a la separación entre grupos. Para todos los análisis se usó el programa Stata 7 (StataCorp., 2001).
2. Anatomía macroscópica, densidad relativa $\left(\rho_{\mathrm{r}}\right)$ y contenido de humedad (C.H.).

En el predio El Grande se seleccionaron y derribaron tres árboles de diámetro no mayor a $20 \mathrm{~cm}$, de los que se obtuvieron tablillas longitudinales de $15 \mathrm{~cm} \times 7 \mathrm{~cm} \times 1 \mathrm{~cm}$, para la determinación de las características macroscópicas (tipo de hilo, textura y veteado), de acuerdo con Tortorelli (1956), y el color con las Tablas de Munsell (Munsell Color Co., 1954). Adicionalmente, a una altura de $1,30 \mathrm{~m}$ se cortaron, hacia el ápice del individuo, tres rodajas de $5 \mathrm{~cm}$ de grueso para muestrear al azar cubos de $3 \mathrm{~cm}$ de lado abarcando toda la superficie transversal.

A los cubos se les determinó volumen en verde (por desplazamiento de agua), peso fresco y peso anhidro (peso seco constante) para calcular densidad relativa y C.H. al corte. Para tal efecto se emplearon las ecuaciones 4 y 5 respectivamente.

$$
\rho_{r}=\frac{p a}{w}
$$

donde:

$\rho_{r}: \quad$ densidad relativa

pa: peso anhidro

vV: peso del volumen desplazado.

$$
C . H .=\left(\frac{p v-p a}{p a}\right) 100
$$

donde

pv: peso fresco de las muestras pa: peso anhidro de las muestras 
La clasificación de las características de la madera se hizo de acuerdo con la IAWA (1989). Se estimaron las medias y las desviación estándar de estos caracteres.

3. Pruebas tecnológicas: secado, contenido de humedad de $12 \%\left(\mathrm{C} . \mathrm{H}_{12} \%\right)$, contracciones, cepillado, clavado, ensamblado y torneado. Elaboración de objetos.

Estas pruebas se realizaron para demostrar que la madera de $T$. micrantha, si es secada al aire libre y cuidada en el proceso, puede ser una alternativa para producir objetos de uso rural a bajo costo, con herramientas manufacturadas por los mismos productores. Las pruebas tecnológicas se efectuaron en la UNAM-I, en una región donde el contenido de humedad en equilibrio es cercano a $12 \%$.

De los árboles derribados se elaboraron tablones de $30 \mathrm{~cm}, 60 \mathrm{~cm}$ y $80 \mathrm{~cm}$ de largo y $5 \mathrm{~cm}$ de grosor, que fueron las dimensiones alcanzadas debido al diámetro. Del tablón de $30 \mathrm{~cm}$ se obtuvieron 10 cubos de $5 \mathrm{~cm}$ de arista para pesarlos en fresco. Simultáneamente, los cubos y los tablones se dejaron secar al aire libre, bajo techo. Diariamente los cubos se pesaron hasta que alcanzaron un peso constante. Los cubos se emplearon para determinar la contracción volumétrica, registrándose los cambios en las dimensiones transversales $y$ longitudinal, cuando se alcanzó el peso anhidro al secarlos en un horno. Cuando los cubos alcanzaron el $\mathrm{C} . \mathrm{H}_{12 \%}$, se presumió que también lo hicieron las tablas; esto se corroboró con un espécimen de cada tablón, encontrándose similitudes en los C.H. Para el cálculo de esta variable, se aplicó la ecuación 5 . Se hizo un ANOVA para observar diferencias entre la densidad relativa, los contenidos de humedad y las contracciones.
Con las tablas al $12 \%$ de C.H., se hicieron pruebas de cepillado, clavado, ensamblado y torneado. Se manufacturaron varios objetos: un banco, varios percheros y objetos torneados como patas de mesa. Las pruebas de cepillado consistieron en pasar sobre las superficies longitudinales al hilo y en contra una garlopa o cepillo de madera rudimentario. El clavado consistió en introducir clavos de varios tamaños a través de superficies transversales y longitudinales. Se hicieron tres tipos de ensamblado: de cruz, de 45 grados y de espiga con escolopo. Para ello se utilizaron herramientas como gurbias rudimentarias, formones y serruchos pequeños. El torneado se probó en un taller local de carpintería para mostrar su respuesta al astillamiento y estabilidad como pieza de soporte. Se elaboraron objetos de pequeño formato para poder ser transladados.

Los resultados de las pruebas de uso en carpintería se llevaron a la comunidad de productores en donde se desarrollaron talleres de carpintería en varias localidades del municipio de Pahuatlán, en la Sierra Norte de Puebla.

\section{RESULTADOS}

\section{Anatomía de la madera de T. micrantha}

\section{Características macroscópicas.}

La madera no presenta zonas de crecimiento definidas, ni diferencias entre albura y duramen. Es de color castaño claro (7,5 YR 6/4), el hilo es entrecruzado, la textura es mediana marcada por los vasos, el veteado suave, no presenta olor ni sabor. No presenta anillos o zonas de crecimiento. Los vasos son visibles a simple vista, el parénquima axial y radial sólo son visibles con lupa (Fig. 2). 


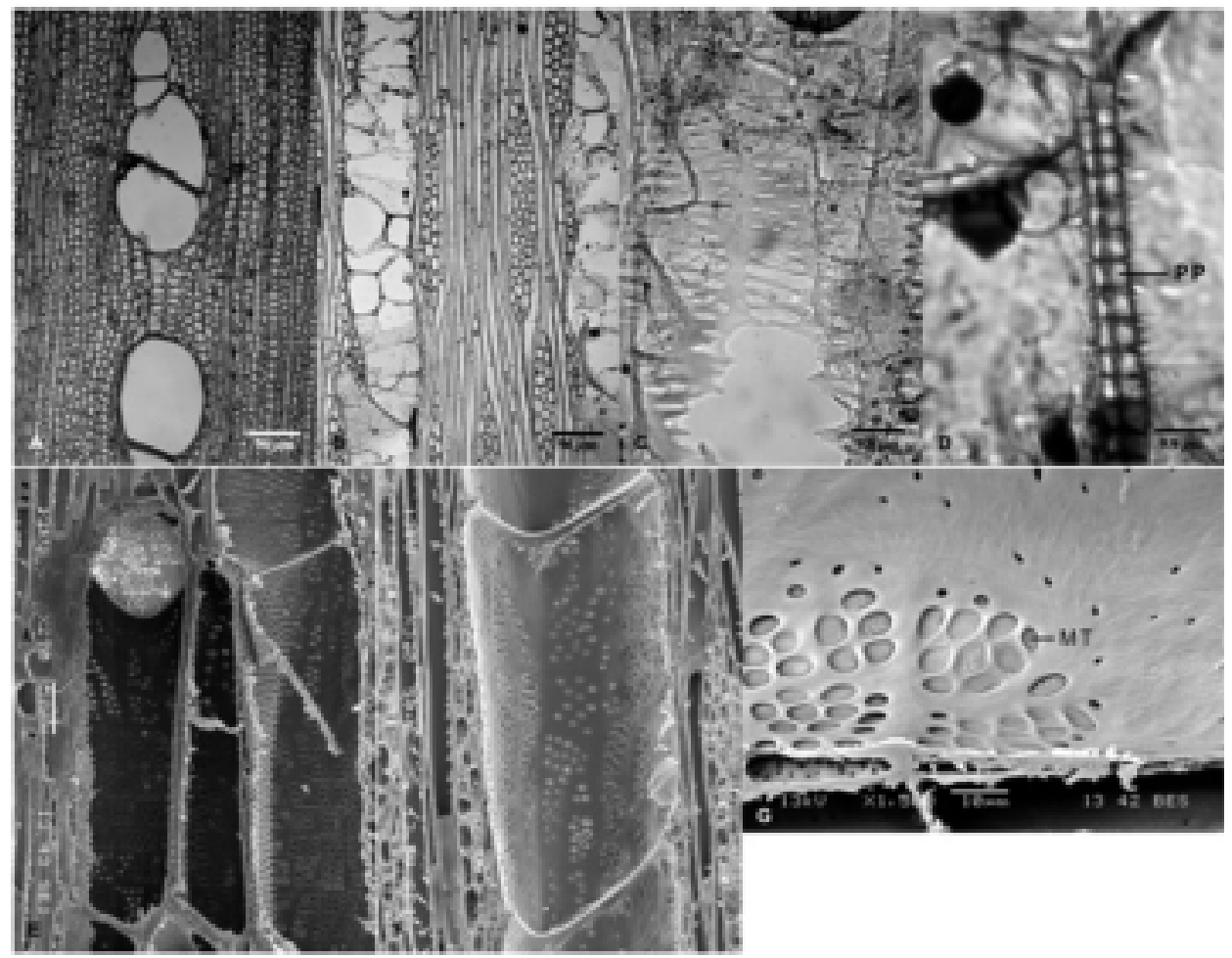

Figura 2. Características anatómicas. A-C. Secciones transversal, tangencial y radial. D. Detalle de la conexión lateral entre dos punteaduras intervasculares. E y F. Vasos. Detalles de las punteaduras y campos de cruzamiento. G. Punteaduras de campo de cruzamiento. PP. Pared de la punteadura. MT. Membrana de la tílide.

Características microscópicas (Fig. 2A-G, Tabla1).

La porosidad es difusa, los elementos de vaso son múltiples radiales de dos a cuatro, solitarios, moderadamente pocos $7(3-10) / \mathrm{mm}^{2}$, medianos en longitud, 556 (353-759) $\mu \mathrm{m}$ y de diámetro tangencial mediano 197 (150-240) $\mu \mathrm{m}$. Las punteaduras intervasculares son areoladas alternas y las de campo de cruzamiento vaso-radio tienen rebordes internos reducidos pero distinguibles. Las punteaduras de campo miden de alto 7,5 $(6-8,2) \mu \mathrm{m}$ y de ancho $12(10-15) \mu \mathrm{m}$ y las intervasculares miden de alto $7(6-8) \mu \mathrm{m}$ y de ancho $11(10-12,4) \mu \mathrm{m}$; se presentan tanto en caras radiales como tangenciales, ambos tipos se consideran medianas. La conexión de dos punteaduras areoladas en sección lateral, se aprecia como un disco central con dos estructuras tubulares hacia las cavidades de los vasos (Fig. 2D). De frente, la apertura interna de estas punteaduras es lisa (Fig. 2F); la conexión entre las punteaduras de vaso-parénquima sólo muestra una cavidad en semidisco. Las cavidades de los vasos se bloquean con tílides de paredes delgadas. Las tílides provienen del parénquima axial. 
Tabla 1. Promedio y desviación estándar de los caracteres microscópicos.

\begin{tabular}{lcccc}
\hline Carácter & Localidad 1 & Localidad 2 & Localidad 3 & Localidad 4 \\
\hline Vasos/mm & 7 & 6 & 6 & 9 \\
& $( \pm 3)$ & $( \pm 2)$ & $( \pm 2)$ & $(c 3)$ \\
*Diámetro vasos & 191 & 198 & 202 & 203 \\
& $( \pm 36)$ & $( \pm 34)$ & $( \pm 40)$ & $(+38)$ \\
*Longitud vasos & 491 & 626 & 526 & 578 \\
& $( \pm 200)$ & $( \pm 197)$ & $( \pm 177)$ & $( \pm 191)$ \\
& 50 & 51 & 56 & 57 \\
*Anchura radios & $( \pm 12)$ & $( \pm 14)$ & $( \pm 15)$ & $( \pm 11)$ \\
& 324 & 353 & 331 & 352 \\
*Altura radios & $( \pm 68)$ & $( \pm 70)$ & $( \pm 54)$ & $( \pm 58)$ \\
& 6 & 6 & 6 & 5.5 \\
\# de radios/mm & $( \pm 1,3)$ & $( \pm 1,5)$ & $( \pm 1,4)$ & $( \pm 1,5)$ \\
& 24,8 & 25,5 & 21,7 & 21,5 \\
*Diámetro fibras & $( \pm 5,6)$ & $( \pm 4,6)$ & $( \pm 3,5)$ & $( \pm 4)$ \\
& 1,74 & 1,059 & 1,69 & 1,430 \\
*Grosor pared fibras & $( \pm 0,5)$ & $( \pm 0,4)$ & $( \pm 0,4)$ & $( \pm 0,4)$ \\
& 1149 & 1040 & 1007 & 990 \\
*Longitud fibras & $( \pm 247)$ & $( \pm 190)$ & $( \pm 275)$ & $( \pm 169)$ \\
\hline
\end{tabular}

*valores en micras.

El parénquima axial es vasicéntrico de una hilera de células. En caras tangenciales se presentan en cordones de más de 8 células de longitud. Presenta muchos almidones hacia la periferia $y$ algunos contenidos de origen polifenólico hacia el centro.

Los radios son triseriados $3(1-4)$ series, angostos $53(41-78) \mu \mathrm{m}$, bajos 340 (230-441) $\mu \mathrm{m}$ y moderadamente numerosos $6(5-7) / \mathrm{mm}$. Contienen almidones en albura y pocos contenidos de origen polifenólico hacia el centro.

Las fibras son de tipo libriforme, de longitud mediana 1047 (839-1254) $\mu \mathrm{m}$, de diámetro fino $23(18-28) \mu \mathrm{m}$ y paredes gruesas 2(1-3) $\mu \mathrm{m}$. No presentan contenidos de ningún tipo.

\section{Variación anatómica y correlaciones}

Los ANOVA de las características anatómicas señalan que el único carácter similar entre individuos y poblaciones fue el grosor de la pared de los vasos y el resto fue significativamente diferente (Tabla 2).

Para los índices mecánicos el I.C. de las fibras fue similar entre individuos y el I.C. de vasos entre poblaciones. Los índices hidráulicos son significativamente diferentes entre individuos y entre poblaciones (Tabla 3).

Las correlaciones ( $r$ ) entre las características anatómicas $(p<0,05)$ fueron: 
Tabla 2. ANOVA de las características anatómicas, mecánicas e hidráulicas, entre árboles y entre poblaciones

\begin{tabular}{lcccccc}
\hline & \multicolumn{3}{c}{ Árboles } & \multicolumn{3}{c}{ Poblaciones } \\
Carácter & $R 2$ & $F$ & $P$ & $R 2$ & $F$ & $P$ \\
\hline & & & & & & \\
poros/mm2 & 0,0349 & 5,0152 & $<0,01$ & 0,1844 & 62.92 & $<0,01$ \\
Diámetro vasos & 0,0812 & 12.25 & $<0,01$ & 0,0136 & 3.849 & $<0,01$ \\
Longitud vasos & 0,357 & 5.138 & $<0,01$ & 0,062 & 18.603 & $<0,01$ \\
Grosor pared vaso & $<0,01$ & 0,0001 & 1 & $<0,01$ & 0,0001 & 1 \\
Anchura radios & 0,1523 & 20,76 & $<0,01$ & 0,0434 & 10,53 & $<0,01$ \\
Altura radios & 0,0706 & 8.777 & $<0,01$ & 0,016 & 3,780 & 0,01 \\
\# de radios/mm & 0,0758 & 9,465 & $<0,01$ & 0,0399 & 9,629 & $<0,01$ \\
Diámetro fibras & 0,0204 & 4,89 & $<0,01$ & 0,136 & 73.98 & $<0,01$ \\
Grosor pared fibras & 0,0197 & 4.731 & $<0,01$ & 0,322 & 223.25 & $<0,01$ \\
Longitud fibras & 0,0605 & 15,122 & $<0,01$ & 0,091 & 47.0956 & $<0,01$ \\
I.V. & 0,0451 & 6,551 & $<0,01$ & 0,0769 & 23,20 & $<0,01$ \\
I.C vasos & 0,0301 & 4.309 & $<0,01$ & 0,0057 & 1.61 & 0,184 \\
I.C fibras & 0,0077 & 1.8256 & 0,0907 & 0,2547 & 160 & $<0,01$ \\
Kh -(vaso) (10-11) & 0,0861 & 13,07 & $<0,01$ & 0,0281 & 8,057 & $<0,01$ \\
Kh -área (10-09) & 0,0558 & 8,198 & $<0,01$ & 0,0986 & 30,471 & $<0,01$ \\
\hline & & & & & & \\
\hline
\end{tabular}

Tabla 3. Promedio y desviación estándar de los índices mecánicos e hidráulicos.

\begin{tabular}{|c|c|c|c|c|}
\hline Índice & Localidad 1 & Localidad 2 & Localidad 3 & Localidad 4 \\
\hline Í. C vasos & $\begin{array}{c}0,0023 \\
( \pm 0,003)\end{array}$ & $\begin{array}{c}0,0019 \\
( \pm 0,0011)\end{array}$ & $\begin{array}{c}0,0021 \\
( \pm 0,0021)\end{array}$ & $\begin{array}{c}0,0029 \\
( \pm 0,0018)\end{array}$ \\
\hline I. C fibras & $\begin{array}{c}0,034 \\
( \pm \quad 0,025)\end{array}$ & $\begin{array}{c}0,0102 \\
( \pm 0,0111)\end{array}$ & $\begin{array}{c}0,0461 \\
( \pm 0,031)\end{array}$ & $\begin{array}{c}0,056 \\
( \pm 0,042)\end{array}$ \\
\hline $\begin{array}{l}K_{h-v a s o} \\
(10-11)\end{array}$ & $\begin{array}{c}3,93 \\
( \pm 2,43)\end{array}$ & $\begin{array}{c}4,44 \\
\pm 2,72)\end{array}$ & $\begin{array}{c}5,34 \\
( \pm 4,01)\end{array}$ & $\begin{array}{c}5,05 \\
( \pm 3,4)\end{array}$ \\
\hline $\begin{array}{l}\mathrm{K}_{\mathrm{h} \text {-área }} \\
(10-09)\end{array}$ & $\begin{array}{c}2,32 \\
( \pm 2,88)\end{array}$ & $\begin{array}{c}1,6 \\
( \pm 1,5)\end{array}$ & $\begin{array}{c}2,72 \\
( \pm 4,07)\end{array}$ & $\begin{array}{c}5,3 \\
( \pm \quad 6,69)\end{array}$ \\
\hline $\mathrm{ka} / \mathrm{kv}$ & 59 & 36 & 50 & 104 \\
\hline
\end{tabular}


El diámetro y la longitud de vaso $(r=-0,06)$; el diámetro de vaso y la anchura de radio $(0,11)$. El número de poros con la longitud de vaso $(0,10)$ y ésta última con la anchura de radio $(0,10)$. El diámetro de fibras con el diámetro de vaso $(0,10)$, con la longitud de vaso $(0,14)$ y con la anchura de radio $(0,18)$.

\section{Análisis discriminante y comparaciones múltiples (Tabla 4, Fig. 3)}

En la figura 3 se presenta la dispersión de las 699 muestras de 28 árboles de las 4 localidades en un espacio definido por las dos primeras funciones del análisis discriminante, que explican $89,79 \%$ de la variabilidad observada donde ambas funciones son significativas $(p<0,0001)$. La $\lambda$ de Wilks para las dos primeras funciones es relativamente baja $(0,353)$ pero significativa $(p<0,0001)$. Se puede observar que existe heterogeneidad dentro de las localidades y entre las varia- bles consideradas, sin embargo, algunas localidades se separan claramente entre ellas en el espacio discriminante, tales son los casos de las localidades Bola de Oro y Los Barrales (Fig. 3). La discriminación está dada por las variables "grosor de la pared de las fibras y por el "número de radios" para ambas funciones discriminantes, ya que representa los mayores valores obtenidos en los vectores característicos (eigenvectores) (Tabla 4).

Las comparaciones múltiples de Tukey-Kramer confirman que el "grosor de la pared de la fibra" es la carácter anatómico que separa $\left(R^{2}=0,32\right)$ a la localidad Bola de Oro de las otras tres. En el mismo sentido se confirma que "el número de $\operatorname{radios} / \mathrm{mm}$ " separa a la localidad Los Barrales de las otras tres. Para la mayoría de los caracteres anatómicos, los ANOVA muestran diferencias significativas entre árboles y entre poblaciones, sin embargo, las $\mathrm{R}^{2}$ son muy pequeñas, por lo que las diferencias apenas se perciben.

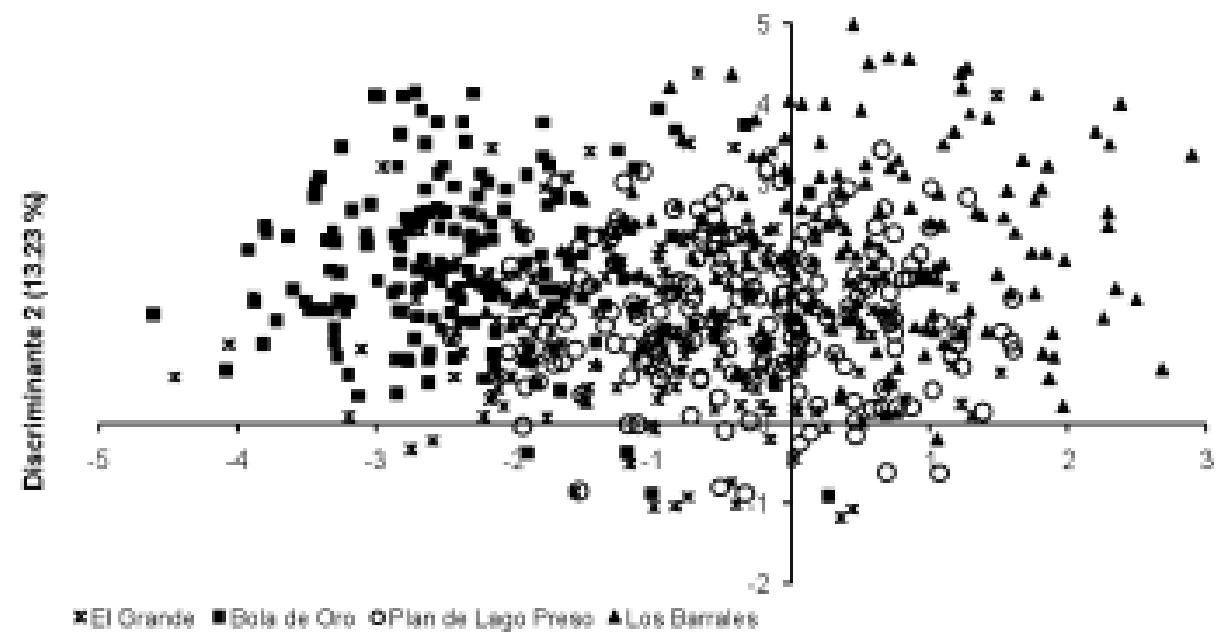

Discriminante $1(76.55 \%$

Figura 3. Diagrama de dispersión de las 699 muestras provenientes de cuatro localidades en un espacio discriminante, definido por las dos primeras funciones del ADM que explican $89,79 \%$ de la variabilidad de la matriz (Tabla 4). 


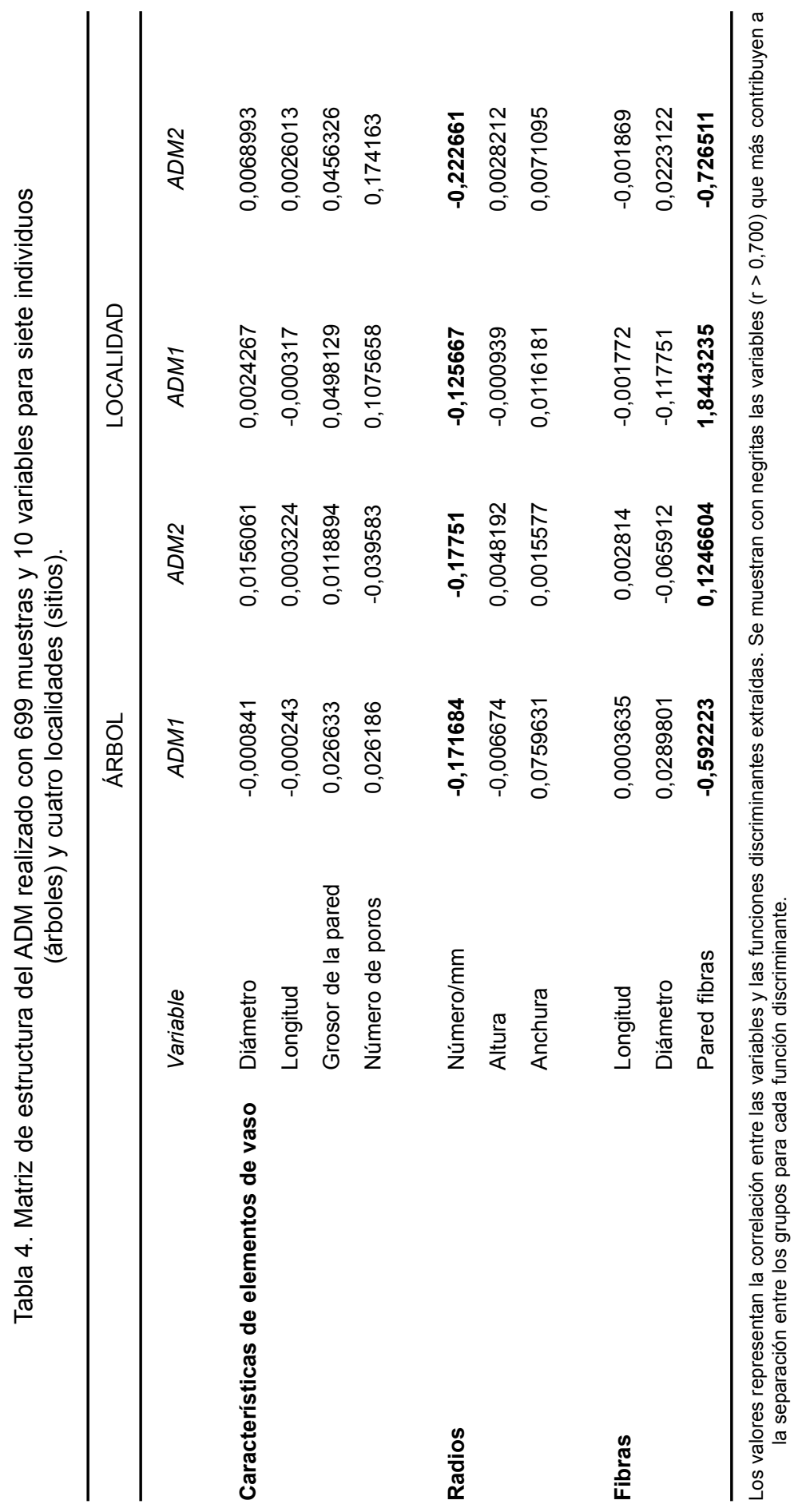




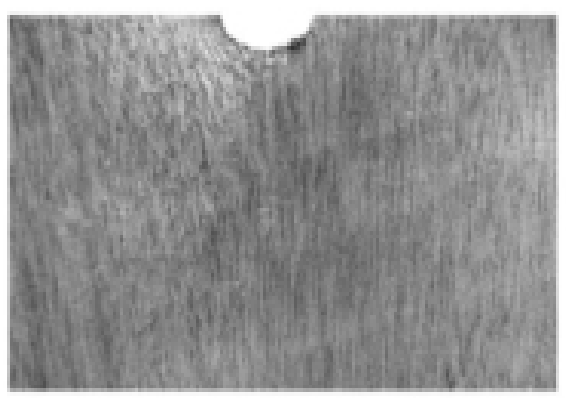

A

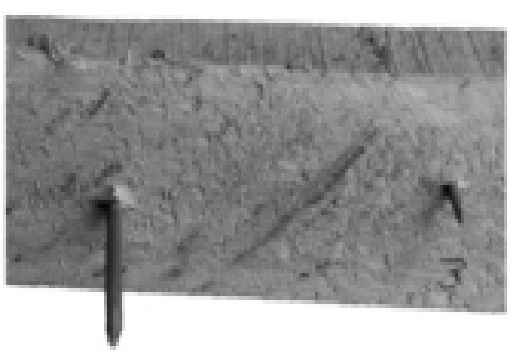

C

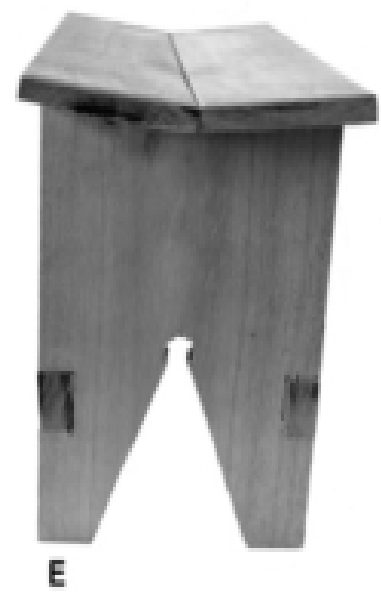

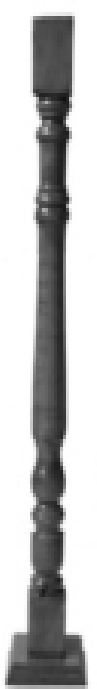

$\mathbf{F}$

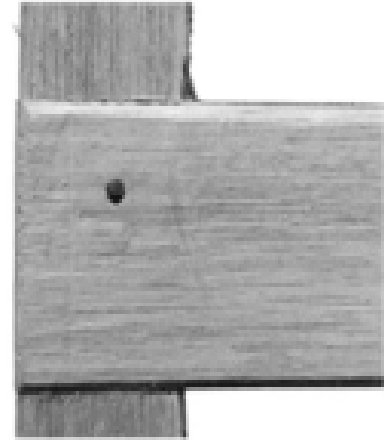

B

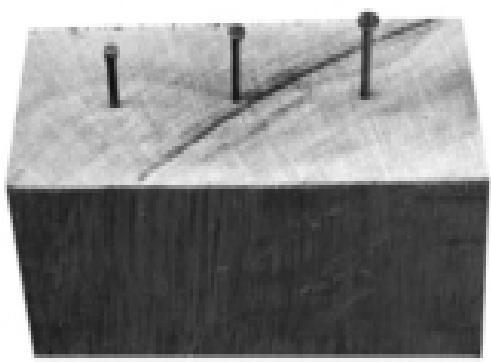

D

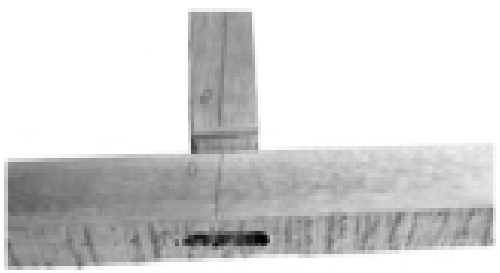

G

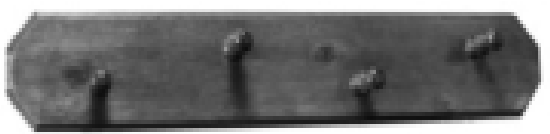

H

Figura 4. Características macroscópicas y pruebas de carpintería A.Textura, hilo, veteado y terminado por cepillado. B-D. Clavado. E-H. Ensamblado y torneado. 


\section{Densidad relativa $\left(\rho_{r}\right)$, contenido de humedad (C. H.) y contracciones}

La densidad relativa fue mediana $(0,453)$. El contenido de humedad en estado verde fue de $48 \%$. El porcentaje de la contracción volumétrica fue 11,47 $( \pm 0,72)$;

Los análisis de varianza mostraron que sólo hubo diferencias significativas en los valores del C.H. de los cubos $\left(R^{2}=0,248 ; F=6,61 ; P<0,01\right)$

\section{Pruebas tecnológicas}

La madera fue fácil de cepillar, la superficie terminada fue suave sin pérdida de madera, levantó fibrosidad contra el hilo, pero se resolvió con lija sin perder material (Fig. 4A). El clavado en los sentidos transversal y axial no produjo rajadura; el clavo quedó sujeto en la estructura del xilema (Figs. 4B-D). Los ensambles, aunque elaborados con herramientas rudimentarias fueron de fácil manufactura y bajo costo, y demostraron que la madera responde bien al pegamento y al clavado en los tres casos de ensambles.

El torneado produjo una pieza estable y sin astillamiento.

\section{DISCUSIÓN}

Las características anatómicas que presenta $T$. micrantha como: porosidad difusa, poros medianos y los valores de I.V. corresponden un xilema mesomórfico (Carlquist 1977). Asimismo, aunado a los valores de I.C.vaso, I.C.fibra y de densidad relativa sugieren una estrategia xilemática de especie de rápido crecimiento que sacrifica masa por volumen (tablas 1, 2 y 3). Esto último también se observa por la presencia de tílides en vasos, que antecede a un fenómeno de cavitación previo al embolismo. Esto significa una reposición continua de vasos nuevos ante un riesgo de disfunción del xilema (Quintanar, 2009 y Quintanar et al., 2009). En este sentido, Sperry (2003) mencionó que la tasa de intercambio de moléculas de agua por una de $\mathrm{CO}_{2}$ es aproximadamente de 300 de agua. Esto se vincula estrechamente con las estrategias hidráulicas de conducción y los valores de las propiedades físicas y mecánicas del xilema que tiene cada especie para fijar este gas y garantizar el crecimiento y producción de madera. Lo anterior significa que el xilema de $T$. micrantha está en continuo requerimiento hídrico para formar masa foliar, que sugiere el ascenso de grandes cantidades de agua. En este sentido, los datos de Ferreira et al. (2009) predicen un sistema vascular secundario de gran capacidad hidráulica, pero de baja densidad debido a la producción de hojarasca y las grandes tallas que alcanzan los árboles de esta especie y su capacidad de intercepción de la luz por volumen foliar. Lo anterior explica la alta productividad de biomasa registrada para esta especie en términos de su tasa fotosintética, asociada a valores altos de índice de área foliar, pues intercepta mayor cantidad de luz que otras especies pioneras (Selaya et al., 2008) y registra la mayor producción de hojarasca, lo que la caracteriza como una especie de rápido crecimiento y no longeva, (Gelder et al., 2006; Selaya et al., 2008; Ferreira et al., 2009 y Markesteijn, et al., 2010).

Variación anatómica. Los ANOVA muestran que aunque las $\mathrm{R}^{2}$ son bajas, en general los caracteres anatómicos son sensibles al efecto de los factores físicos de los sitios de estudio. Resaltan las relacionados con las propiedades hidráulicas que sugieren que el ajuste en las dimensiones y el número de los vasos está relacionado con los requerimientos hídricos propios de esta especie (tablas 2 y 3 ). Las correlaciones muestran que entre más 
anchos son los vasos, la longitud es menor, aunque esta tendencia sólo se explica en un $6 \%$. Se observa que también los radios son caracteres sensibles, aunque la anchura de estos es el rasgo más correlacionado con los vasos y las fibras. Es decir, a mayor diámetro y longitud de vaso, la anchura de los radios se incrementa, aunque este incremento sólo se explica en $10 \%$ a $11 \%$. Las dimensiones de las fibras son tan sensibles como los elementos de vaso y responden de manera correlacionada con el diámetro y longitud de vasos y con la anchura de radio. Es decir, conforme incrementan las dimensiones de las fibras incrementan los otros caracteres. Sin embargo, estos incrementos en las dimensiones sólo se explican en 10\%, $14 \%$ y $18 \%$, respectivamente. Los análisis ANOVA, de ADM y de TukeyKramer muestran que las localidades Los Barrales y Bola de Oro se separan por caracteres no relacionados con estrategias hidráulicas o de densidad (tablas 2, 3 y 4 y Fig. 3).

\section{Índices mecánicos e hidráulicos}

Se ha señalado que los índices mecánicos de vasos y de fibra que dependen del grosor de sus paredes, miden la resistencia a la implosión cuando estas células están bajo funcionamiento (Hacke et al., 2001; Baas et al., 2004). En esta especie ambos tipos celulares presentan índices muy bajos, lo que está asociado a la densidad relativa característica de $T$. micrantha (Hacke et al., 2001; Gelder et al., 2006; Swenson y Enquist, 2007; Bárcenas y Ordóñez, 2008). Los valores de I.C., predicen una deformación de las pared de los vasos suficiente para colapsarlos, lo que se relaciona con los datos de Selaya et al. (2008) que encuentran que esta especie desarrolla su dosel con mayor velocidad que otras especies pioneras. $\mathrm{Si}$, como señalan estos autores, la tasa evaporativa es alta, entonces la tasa de colapso de vasos podría ser también alta, lo que también explica la presencia de grandes cantidades de tílides incluso cercanas al cambium vascular, que se producen después de un evento de embolismo. Este comportamiento también se observó en el xilema de Gliciridia sepium (Quintanar, 2009).

En Quintanar et al. (2009) se argumenta que en G. sepium el índice de vulnerabilidad está representando pocos vasos en relación con el número/ $/ \mathrm{mm}^{2}$, de manera que si un vaso cavita debido a su diámetro, entonces el área conductora del xilema se reduce. Por otro lado, esta planta tiene vasos medianos en promedio $(180 \mu \mathrm{m})$, valores altos de densidad relativa $(0,82)$, es de lento crecimiento y tiene bajos potenciales hídricos ( $\Psi=-2,4 \mathrm{MPa}$ ) en sitios tropicales secos o perturbados (Quintanar, 2009). Al respecto Markesteijn et al. (2010) registran los valores de potenciales hídricos del suelo $(\Psi=-0.16$ $\mathrm{MPa}$ ) y de las hojas de $T$. micrantha $(\Psi=-1.67 \mathrm{MPa})$ de un bosque tropical lluvioso boliviano, lo que significa que hay un esfuerzo de ascenso de agua de esta especie mucho menor que en $G$. sepium. El xilema de estas especies es similar en el tamaño de vasos y el número de poros y diferentes en sus densidades relativas, sin embargo, en ambas se considera mesomórfico. El valor del estatus hídrico del xilema podrá revelar la mejor compresión de la respuesta del xilema de $T$. micrantha en relación con el uso del agua, su velocidad de crecimiento $y$ longevidad.

Los valores de $\mathrm{K}_{h}$ área $/ \mathrm{K}_{\mathrm{h}}$ vaso en T. micrantha son del orden de 63 (36-104) que, comparado con los de G. sepium (5) Prosopis laevigata (67) o Piptadenia flava (183), procedentes de selvas secas de Veracruz y Oaxaca (Quintanar et al., 2009), sugieren el impacto del número de 
vasos en el flujo másico del agua, considerando las diferencias de los parámetros físicos de los sitios en los que habita. Al comparar las precipitaciones registradas para ambas localidades, se observa que en el municipio de Coatepec (El Grande) se registra mayor precipitación pluvial que en el municipio de Xico (Bola de Oro localidad, Plan de Lago Preso y Los Barrales). De acuerdo con los análisis estadísticos (tablas 1, 2, 3 y 4) y los datos de precipitación y temperatura de las localidades estudiadas, es posible que las variables que componen el medio físico (Gobierno del Estado de Veracruz, 2008) no se vean tan marcadas en la estructura anatómica e hidráulica. De cualquier forma se sugiere que en estudios futuros se correlacionen las características de la madera estudiada con estudios hidrológicos y climáticos.

La transferencia de los resultados a las comunidades rurales cafetaleras en la Sierra Norte de Puebla, detectó un interés en la comunidad para que se desarrollaran talleres continuos para el procesamiento de la madera de T. micrantha. Se destacan las siguientes características de esta madera:

La madera de T. micrantha de especímenes jóvenes presentó características de cepillado que resultaron en un terminado fibroso, pero con el lijado se lograron superficies más suaves (Fig. 4B). Aunque la característica fibrosa puede ser un defecto por el rápido crecimiento de la madera, resaltan las cualidades de no rajarse ni producir astillas Esto la hizo fácil para ensamblar, clavar y tornear (Fig. 4B-D). Por sus características estéticas se sugirió como sustituta de especies como la caobilla, el cedro rosado o especies de mediana densidad, reconociendo que no presenta las propiedades mecánicas o de resistencia para usos en los que se requiere más que una buena estética.
Debido al rápido crecimiento que registra esta especie, la densidad y la estructura celular están asociadas a los valores altos de contracción (Bárcenas y Ordóñez 2008) por lo que se abren las posibilidades para introducir tecnología de secado para áreas rurales que permitan controlar las deformaciones derivadas de esta propiedad. A pesar de esto, los objetos terminados tuvieron características que los campesinos cafetaleros supieron apreciar. En condiciones de buen secado fue fácil de tallar, por lo que se recomendó para esculturas, artesanías, para muebles de todo tipo, caseros, individuales, para oficina o como ornamento y bancos para escuelas principalmente mobiliario de nivel preprimaria (figs. 4G-H). Su color claro aceptó tintes y barnices que pueden adaptarse para cualquier uso. Por sus resultados en la talla y el torneado se recomendó en la elaboración de patas de mesas, soportes de perchero, agarraderas o jaladeras, balastros para barandales de escaleras o redondeo para palos de escoba y juguetes.

Aunque Gelder et al. (2006) y Bárcenas y Ordónez (2008) mencionan que no es resistente a varias pruebas, se recomendó en la elaboración de marcos para puertas, ventanas, bastidores, cuadros sencillos, cuadros de serigrafía, base de mesas. También para molduras, ensambles de zoclos o rodapiés.

\section{CONCLUSIONES}

Trema micrantha presenta un xilema poco resistente a la cavitación, como lo sugieren los bajos valores de I.C.fibra e I.C.vaso asociado a altos valores de I.V.; las densidades bajas y las altas contracciones. Asimismo, los valores de $\mathrm{K}_{\text {harea }} / \mathrm{K}_{\text {hvaso }}$ muestran que los flujos másicos de agua son abundantes, lo que la caracterizaría como una especie pionera. Presentó dife- 
rencias significativas de sus características anatómicas entre árboles y poblaciones, pero sus valores de $\mathrm{R}^{2}$ fueron muy pequeños, lo que sugiere un estudio sobre el impacto del medio físico en los valores anatómicos asociados a las características hidráulicas. Los análisis de comparación múltiple y de ADM, por su sensibilidad, mostraron que el grosor de la pared y el número de radios separaron a las localidades Bola de Oro y Los Barrales de las otras. Los resultados de las propiedades de secado, clavado, ensamblado y torneado sugieren una asociación con el rápido crecimiento y la baja longevidad de esta especie que caracteriza a su xilema, con resultados potenciales hacia usos diversos que pueden beneficiar a las poblaciones rurales cafetaleras, por lo que resulta una especie que puede ser promovida en planes de reforestación o restauración ecológica y ser una alternativa en la economía rural de estas regiones de México.

\section{AGRADECIMIENTOS}

Los autores agradecen al señor ebanista David García por las pruebas tecnológicas y las demostraciones de la madera para la carpintería en las zonas rurales. A los pobladores de Loma Bonita de la Sierra Norte de Puebla que permitieron las demostraciones y a los árbitros anónimos por sus acertadas observaciones. Esta investigación fue financiada por la División de Ciencias Biológicas y de la Salud de la Universidad Autónoma Metropolitana-Iztapalapa y por el Centro de Investigaciones Tropicales de la Universidad Veracruzana.

\section{REFERENCIAS}

Amorim, I.L., A.C. Davide, M.F. Chaves M. 1997. Morfologia do fruto e da semente, e germinação da semente de
Trema micrantha (L.) Blum. Revista Cerne, Lavras 3(1):129-142.

Ackerly, D., S 1997. Trema micrantha (capulín). In: E. González, R. Dirzo y C.R. Vogt. eds. Historia natural de Los Tuxtlas. Conabio-UNAM. México. p:156-58.

Baas, P., F.W. Ewers, S.D. Davis y E. Wheeler. 2004. Evolution of xylem physiology. In: The evolution of plant physiology. Great Britain. Elsevier. p:275-295.

Bárcenas Pazos, G.M y V.R. Ordóñez Candelaria. 2008. Calidad de la madera de los árboles de sombra. In: R. H. Manson, V. Hernández-Ortiz, S. Gallina y K. Mehltreter, eds. Agroecosistemas cafetaleros de Veracruz: Biodiversidad, Manejo y Conservación. INE-Inecol, México, D.F. p:235-246.

Carlquist, S. 1977. Ecological factors in wood evolution: a floristic approach. Amer. J. Bot. 64(7):887-896.

Carvalho, P.E.R. 1994. Espécies florestais brasileiras: recomendações silviculturais, potencialidades e uso da madeira. Colombo: EMBRAPA/CNPF; Brasília: EMBRAPA/SPI. 640 p.

Chattaway, M. 1932. Proposed standards for numerical values used in describing woods. Trop. Woods 29: 20-28.

Ferreira, M.B., V. Gomes y M. Losada. 1976 Subsídios para o estudo de Trema micrantha (L.) Blume. Cerrado, Brasilia. 8(32):30-34.

Ferreira, G.B., G.C. Coelho, J. Schimer y O.A. Lucchese. 2009. Dendrometry and litterfall of neotropical pioneer and early secondary tree species. Biota Neotrop. 9(1):65-71. 
Gómez, C.M.1991. Atlas climático del municipio de Xico. Serie Estudios Climáticos núm. 7. Instituto de Ecología, A.C. 47 p.

Gobierno del Estado de Veracruz. 2008. Municipio de Coatepec. http://portal. veracruz.gob.mx/portal/page?_pageid $=315,3923649 \&$ dad=portal\&_schem a=PORTAL. Febrero 2012.

Gelder, H.A., L. Poorter y F.J. Sterck. 2006. Wood mechanics, allometry, and lifehistory variation in a tropical rain forest tree community. New Phytologist 171:367-378.

Gutiérrez, C.L. y L.J. Dorantes. 2004. Especies forestales de uso tradicional del estado de Veracruz. Potencialidades de especies con uso tradicional del estado de Veracruz, como opción para establecer Plantaciones Forestales Comerciales. Conafor-Conacyt-UV. http://www.verarboles.com/lzpepe/ izpepe.html. Febrero 2012.

Hacke, U.G., J. Sperry, W.T Pockman, S.D. Davis y K.A. McCulloh. 2001. Trends in wood density and structure are linked to prevention of xylem implosion by negative pressure. Oecologia 126:457-461.

IAWA Committee.1989. IAWA list of microscopic features for hardwood identification. IAWA Bull. n. s. 10(3):219-332.

Johansen, D.A. 1940. Plant microtechnique. McGraw Hill. Nueva York. 523 p.

López, C. 2003. The endurance of Mexican Amate paper: Exploring additional dimensions to the sustainable development concept. Tesis doctoral. CSTMTwente University Press. Países Bajos. $330 \mathrm{p}$.

López, C., A. Quintanar-Isaías y M. Vander Meeren. 2012. Mexican bark paper: Evidence of history of tree species used and their fiber characteristics. Economic Botany. 66:138-148.

Markesteijn, L.J. Iraipit, F. Bongers y L. Pooter. 2010. Seasonal variation in soil and plant water potentials in Bolivian tropical moist and dry forest. Journal of Tropical Ecology 26:497-508.

Martínez, M.A., V. Evangelista, F. Basurto, M. Mendoza y A. Cruz-Rivas. 2007. Flora útil de los cafetales en la Sierra Norte de Puebla, México. Revista Mexicana de Biodiversidad 78(1): 15-40.

Munsell Color Company. 1954. Munsell soil color charts. Baltimore. Maryland. $17 \mathrm{p}$.

Melgar, W. 2003. Estado de la diversidad biológica de los árboles y bosques de Guatemala. Documentos de Trabajo: Recursos Genéticos Forestales. FGR/53S Servicio de Desarrollo de Recursos Forestales, Dirección de Recursos Forestales, FAO, Roma, inédito. http://www.fao.org/docrep/ 007/j0605s/j0605s00.htm. Febrero 2012.

National Academy of Sciences. 1980. Trema species. In: Firewood Crops. Shrub and tree species for energy production. Report of an Ad Hoc Panel of the Advisory Committee on Technology Innovation Board of Science and Technology for International Development Commission on International Relations. p:68-69

Quintanar-Isaías, A., J.L. Zárate Castrejón, C. López, e I.H. Salgado-Ugarte. 2004. Anatomía e histoquímica de la corteza de cinco especies de Moraceae. Polibotánica 17:15-38.

Quintanar I.A. 2009. Efecto de la inducción de tílides por cavitación en la arquitectura hidráulica del cocuite, Gliricidia 
sepium (Fabaceae). Tesis de Doctorado. UAM. $146 \mathrm{p}$.

Quintanar I., A., G. Ángeles-Álvarez y J.A. Zavala-Hurtado. 2009. Anatomía, índices físicos e hidráulicos de la madera de Gliricidia sepium (Jacq.) Steud. Madera y Bosques 15(2):71-91.

Quintanar I., A., M.B. Jarquín Pacheco, A.T. Jaramillo Pérez, G. Gutiérrez P., J. Sepúlveda S. y M.A. Jacobo V. 2010. Procesos limpios para el ablandamiento de fibras. San Pablito. Producción sustentable de papel amate en la Sierra Norte de Puebla. Informe Técnico Final. UAM, Fonart, IAIP, UV. $52 \mathrm{p}$.

Selaya G., R.J. Oomen, J.J.C. Netten, M.J.A. Werger y N.P.R. Anten. 2008. Biomass allocation and leaf life span in relation to light interception by tropical forest plants during the first years of secondary succession. Journal of Ecology 96:1211-1221.

Sangalli, A., M.C. Vieira y N.A.H. Zárate. 2002. Levantamento e caracterização de plantas medicinais nativas com propriedades medicinais em fragmentos florestais e de cerrado, em Dourados-MS, numa visão etnobotânica. Acta Horticulturae, The Hague (569):173-184.

Sperry, J.S. 2003. Evolution of water transport and xylem structure. Int. J. Plant Sci. 164(3 Suppl.):S115-S127.

StataCorp. 2001. Stata Statistical Software: Release 7.0. College Station, TX: Stata Corporation.
Streeter, V.L. y Wyllie, E.B. 1985. Fluid mechanics. McGraw Hill. Nueva York. $560 \mathrm{p}$.

Swenson, N.G. y B.J. Enquist. 2007. Ecological and evolutionary determinants of a key plant functional trait: wood density and its community-wide variation across latitude and elevation. American Journal of Botany 94(3): 451-459.

Tatsuoka, M.M. 1970. Discriminant analysis. The study of group differences. IPAT. Champaign, III. $130 p$

Tortorelli, L.A. 1956. Maderas y Bosques Argentinos. ACME. 910 p.

Toledo, M. y J. Salick. 2006. secondary succession and indigenous management in semideciduous forest fallows of the Amazon basin. Biotropica 38(2):1-10.

Vázquez-Yanes, C., A.I. Bátiz M., M.I. Alcocer S., M. Gual D.y C. Sánchez D. 1999. Árboles y arbustos potencialmente valiosos para la restauración ecológica y reforestación. Reporte técnico del proyecto J084. ConabioInstituto de Ecología, UNAM. [en línea]: http://www.conabio.gob.mx/conocimiento/info_especies/arboles/doctos/ indice_especies.html\#Z. Febrero 2012

Williams-Linera, G. 1996. Crecimiento diamétrico de árboles caducifolios $y$ perennifolios del bosque mesófilo de montaña en los alrededores de Xalapa. Madera y Bosques 2(2):53-65. Paz Pérez-Olvera. 2012. La madera de Trema micrantha (L.) Blume de Veracruz, México. Madera y Bosques 18(2):73-91. 